\title{
Ordinary Versus Novel Particle With the Example of Their Spontaneous Transition
}

\author{
Josip Śoln
}

JZS Phys-Tech, Vienna, Virginia 22182, USA

Correspondence: Josip Śoln, JZS Phys-Tech, Vienna, Virginia 22182, USA. E-mail: jurasoln@ yahoo.com

Received: September 28, 2021 Accepted: December 1, 2021 Online Published: December 17, 2021

doi:10.5539/apr.v13n3p15

URL: https://doi.org/10.5539/apr.v13n3p15

\begin{abstract}
The bicubic equation of particle limiting velocity formalism yields three solutions $c_{1}, c_{2}$ and $c_{3}$, (primary, secondary and tertiary) limiting velocities in terms of the congruent parameter $z(m)=3 \sqrt{3} m v^{2} / 2 E$ which is defined in terms of $m, v$, and $E$, respectively being particle mass, velocity and energy. The bicubic equation discriminant $D$ is given in terms of the congruent parameter $z(m)$. When one has $z^{2}(m) \leq 1$ with the discriminant satisfying $D \leq 0$ then we are talking about limiting velocities of ordinary particles. Good examples are the relativistic particles such as electron, neutrino, etc., with luminal limiting velocity $c_{3}=c$ and calculated superluminal $c_{2}$, and imaginary superluminal $c_{1}$, all corresponding to the real particle energy. On the specific level, the situations like these, we discuss in the muon neutrino velocities with the OPERA detector and the electron velocities from the 2010 Grab Nebula Flare. The $z(m)=1$ value separates the ordinary particles from novel particles, associated with $D \geq 0$ and $z^{2} \geq 1$ with new novel particle limiting velocity solutions $c_{1}, c_{2}$ and $c_{3}$ which depend, in addition to $z(m)$, also on the congruent angle $\alpha(m)$, nonlinearly related to $z(m)$. These solutions are discussed on the newly defined sterile neutrino which here is modeled as an ordinary particle with $z^{2} \leq 1$ spontaneously transiting via $z(m)=1$ into the modeled novel sterile neutrino with $z^{2} \geq 1$. All ordinary and novel particles limiting velocities carry real particle energies; the ordinary particle limiting velocity solutions being in quadratic forms, while the novel particle limiting velocity solutions being respectively, in quadratic complex form, linear complex form, and just congruent angle $\alpha$ complex quadratic form.
\end{abstract}

Keywords: cpmplex limiting velocity, real energy, congruent parameter, novel particle

\section{Introduction}

In order to treat the ordinary as well as the novel particles on equal footing, we shall relay on series of articles on bicubic equation limiting particle velocity formalism as developed in (Śoln, J., 2014-2021). Here we start with somewhat amplified particle energy changed and upgraded from usual relativistic kinematics,

$$
E^{2}=\frac{m^{2} c^{4}}{1-v^{2} / c^{2}}, \vec{p}=\frac{E}{c^{2}} \vec{v}
$$

The kinematical change is evident in $(1,2)$ as in (1) positive energy $E$ can exist even if $c^{2}<0$. For a particle of mass $m$ and velocity $\vec{v}$ one writes enlarged "mass=shell" condition

$$
\vec{p}^{2} c^{2}-E^{2}=-m^{2} c^{4}
$$

Relation (3) together with either (1) or (2) yields the particle limiting velocity $c$ bicubic equation (Śoln, J., 2014).

$$
\left(\frac{c^{2}}{v^{2}}\right)^{3}-\left(\frac{E}{m v^{2}}\right)^{2}\left(\frac{c^{2}}{v^{2}}\right)+\left(\frac{E}{m v^{2}}\right)^{2}=0
$$

The three limiting velocity solutions of the bicubic equation (4), $c_{1}, c_{2}$, and $c_{3}$, respectively the primary, secondary and tertiary, in general will depend on $m, v$ and $E$. The solutions are characterized by the discriminant $D$ together with the congruent parameter $z(m)$ : 


$$
\begin{aligned}
D(m) & =\frac{1}{4}\left(\frac{E}{m v^{2}}\right)^{4}\left[1-\frac{4}{27}\left(\frac{E}{m v^{2}}\right)^{2}\right] \\
& =\left(\frac{27}{8}\right)^{2} \frac{1}{z^{4}(m)}\left(1-\frac{1}{z^{2}(m)}\right), \\
E & =\frac{3 \sqrt{3} m v^{2}}{2 z(m)} \\
\text { Ordinary particles } & : \quad D(m) \leq 0, z^{2}(m) \leq 1, \\
\text { Novel particles } & : \quad D(m) \geq 0, z^{2}(m) \geq 1
\end{aligned}
$$

The usual ordinary particles that are otherwise also associated with the relativistic kinematics (Adams, T. et al., 2012; Stecker, F. W., 2015; Rade, L., \& Westergren, B., 1999; Gricths, D., 1987; Eisberg, R. M., 1966), such as electron $e$ and neutrino $v$, belong to $D(m) \leq 0, z^{2}(m) \leq 1$. The specific examples that we discuss briefly are first the muon neutrino velocity with the OPERA detector (Adams, T. et al., 2012; Śoln, J., 2014-2017) with neutrino energy $E(v(\mu)) \simeq 17 \mathrm{GeV}$ and second the 2010 Grab Nebula Flare (Stecker, F. W., 2015; Śoln, J., 2017) with electron energy $E(e) \simeq 5.1 P e V$. As the example of the novel particle, that belongs to $D(m) \geq 0, z^{2}(m) \geq 1$, we take the K. C. Y. Ng et al. (Ng, Kenney C. Y., et al., 2019) sterile neutrino $\chi$ of $m_{\chi} \geq 12 \mathrm{keV} / \mathrm{c}^{2}$ which, although in (Ng, Kenney C. Y., et al., 2019) is called dark matter particle, is well suited to belong to novel particle.

Section 2 is devoted to obtaining the ordinary particle exact solutions for squares of limiting velocities $c_{1}^{2}, c_{2}^{2}$ and $c_{3}^{2}$, respectively of the primary, secondary and tertiary, satisfying $D<0$ and $z \leq 1$. In this section, the relationship between the usual relativistic particle velocity $\beta=v / c$ and the congruent parameter $z$ is established, with $c$ the velocity of light. It is also noticed that as long as $z$ has fixed value, the particle energy remains the same, regardless which limiting velocity it follows. This was demonstrated on the cases of OPERA detector of muon neutrino velocity (Adams. T. et al.; Śoln, J., 2014-2017) and the 2010 Grab Nebula Flare (Stecker, F. W., 2015; Śoln, J., 2017).

In Section 3 the exact squares of novel particle limiting velocities, $c_{1}^{2}, c_{2}^{2}$ and $c_{3}^{2}$, respectively primary, secondary and tertiary, are given with $D(m) \geq 0, z^{2}(m) \geq 1$ satisfied. Here also the new real congruent angle $\alpha(m)$, with two mutually consistent connections to the congruent parameter $z(m)$ is introduced. Furthermore, here one establishes also three separate rather simple equivalent algebraic relations between $z(m)$ and $\alpha(m)$ which allow eliminating $z(m)$ in favor of $\alpha(m)$ if desired. Otherwise, the $z(m)$ and $\alpha(m)$ will appear together in the limiting velocity solutions .The particle energies,despite limiting velocities involving some imaginary portions, are all real and of the same value, for fixed values of $z(m)$ and related $\alpha(m)$. The equal energies then change with simultaneous changes in $z(m)$ and $\alpha(m)$.

Section 4 is devoted to developing of three linear particle limiting velocities, primary, $c_{1}$, secondary, $c_{2}$, and tertiary, $c_{3}$, from the corresponding quadratic limiting velocity expressions by using the binomial equations (Rade, L., \& Westergren, B., 1999, p. 65).

In Section 5 with the elimination of the congruent parameter $z(m)$, one deals with samples of physical quantities with novel matter particle limiting velocities containing exclusively the congruent angle $\alpha(m)$.

Section 6 introduces the separating congruent parameter point of $z=1$ between the ordinary and novel particles. On the example of sterile muon neutrino, as a novel particle, the evolution to larger values congruent parameter with $z(m) \geq 1$ is carried out. Summarizing the results we end up with speculative assumptions that perhaps some of the called dark matter particles could belong to some novel particles introduced here.

\section{Limiting Velocity Solutions in Quadratic Forms for Real Energy Ordinary Particles}

As indicated in the introduction (5), we start with $D<0$ and $z \leq 1$ for discriminant and the congruent parameter respectively, with assumption to be satisfied with respective mass $m$, velocity $v$ and energy $E$. Then the three squares of particle limiting velocity solutions are from (4) (Śoln, J., 2014-2018):

$$
\begin{gathered}
D \leq 0, z \leq 1, z=\frac{3 \sqrt{3} m v^{2}}{2 E} \\
\frac{c_{1}^{2}(m)}{v^{2}}=\frac{3}{z(m)} \sin \left[\frac{1}{3}\left(\pi-\sin ^{-1}(z(m))\right)\right]>0 ;
\end{gathered}
$$




$$
\begin{gathered}
\frac{c_{2}^{2}(m)}{v^{2}}=-\frac{3}{z(m)} \sin \left[\frac{1}{3}\left(\pi+\sin ^{-1}(z(m))\right)\right]<0 ; \\
\frac{c_{3}^{2}(m)}{v^{2}}=\frac{3}{z(m)} \sin \left[\frac{1}{3} \sin ^{-1}(z(m))\right]>0 .
\end{gathered}
$$

As noted in (Śoln, J., 2021), for $D \leq 0, z \leq 1$, these limiting velocities satisfy a variety of conditions, most of which we enumerate here by utilizing (6.0):

$$
c_{1}^{2}+c_{2}^{2}+c_{3}^{2}=0
$$

Utilizing the Cardano's formula (Rade, L., \& Westergren, B., 1999) on page 56, one also deduces what follows.

$$
\begin{aligned}
c_{1}^{2} c_{2}^{2} c_{3}^{2} & =-\left(\frac{E v}{m}\right)^{2}=-\frac{27 v^{6}}{4 z^{2}}, \\
z^{2}(m)= & \frac{-27 v^{6}}{4 c_{1}^{2} c_{2}^{2} c_{3}^{2}} \\
c_{1}^{2} c_{2}^{2}+c_{1}^{2} c_{3}^{2}+c_{2}^{2} c_{3}^{2} & =-\left(\frac{E}{m}\right)^{2}=-\frac{27 v^{4}}{4 z^{2}}, \\
z^{2}(m) & =\frac{-27 v^{4}}{4\left(c_{1}^{2} c_{2}^{2}+c_{1}^{2} c_{3}^{2}+c_{2}^{2} c_{3}^{2}\right)} \\
c_{1}^{4}+c_{2}^{4}+c_{3}^{4} & =2\left(\frac{E}{m}\right)^{2}=\frac{27 v^{4}}{2 z^{2}}, \\
z^{2}(m) & =\frac{27 v^{4}}{2\left(c_{1}^{4}+c_{2}^{4}+c_{3}^{4}\right)}
\end{aligned}
$$

The resulting relations (7.1)-(7.4) for the congruent parameter $z(m)$ strongly indicate the influence of each limiting velocity on, what we call, the ordinary particles, with given mass $m$, velocity $v$, and the energy $E$ (compare with (6.0)). Some of these limiting velocities $c_{1}, c_{2}$, and $c_{3}$, although related to ordinary particles such as electron and neutrino, practically may be difficult to observe with present day technology. On the other hand, the question also is are all the ordinary particles yet identified. On general grounds, these relations(7.1)-(7.4) are useful to see the importance of all physical quantities that influence the generation of particle limiting velocities.

At this point, it is worth to point the universality of the congruent parameter $z(m)$. In fact knowing the particle energy $E$ is the most important thing to know to get the congruent parameter $z(m)$. We demonstrate this with the usual relativistic energy E(relative) as appearing in (Griфths, D., 1987) and (Eisberg, J. M., 1966). To obtain $z$ (m,relative), we equate $E$ (relative) with our $E$ from (6.0). Here are the details with numerical results in the Table1. to follow:

$$
\begin{aligned}
E(\text { relative }) & =\frac{m v^{2}}{\beta^{2}\left(1-\beta^{2}\right)^{\frac{1}{2}}}=\frac{3 \sqrt{3} m v^{2}}{2 z(m, \text { relative })}: \\
z(m, \text { relative }) & =\frac{3 \sqrt{3}}{2} \frac{\beta^{2}}{\gamma}, \beta=\frac{v}{c}, \gamma=\left(1-\beta^{2}\right)^{-\frac{1}{2}}
\end{aligned}
$$

Table 1: $0<z(m$, relative $)<1 \quad$ versus $\quad 0<\beta<1$

$\begin{array}{cccccccccccc}\beta: & 0 & 0.1 & 0.2 & 0.3 & 0.4 & 0.5 & 0.6 & 0.7 & 0.8 & 0.9 & 1 \\ z(m, \text { relative }): & 0 & 0.026 & 0.1 & 0.223 & 0.38 & 0.56 & 0.6 & 0.9 & 1 & 0.35 & 0\end{array}$


Now one can apply the Table1 to any solution from (6). For instance, choose $\beta \rightarrow 1(v \rightarrow c)$ and from (8.2) $z(m$, relative $) \rightarrow$ 0 . With this, for example from (6.3), staightforwardly one obtains that $c_{3}^{2} \rightarrow c^{2}$. Later, more specific examples follow. The simplest way to evaluate equal numerical energies but associated with different limiting velocities is to evaluate first $v^{2} / z(m)\left(c_{1}^{2}, c_{2}^{2}, c_{3}^{2}\right)$ from solutions (6). These when submitted to the expression from (5) one obtains equal energies with different particle limiting velocities:

$$
\begin{aligned}
& E\left(c_{1}\right)=\frac{3 \sqrt{3} m v^{2}}{2 z(m)}=\frac{3 \sqrt{3} m v^{2}}{2 z(m)}\left(c_{1}^{2}\right)=\frac{\sqrt{3} m c_{1}^{2}}{2 \sin \left[\frac{1}{3}\left(\pi-\sin ^{-1}(z(m))\right)\right]}, \\
& E\left(c_{2}\right)=\frac{3 \sqrt{3} m v^{2}}{2 z(m)}=\frac{3 \sqrt{3} m v^{2}}{2 z(m)}\left(c_{2}^{2}\right)=\frac{\sqrt{3} m\left(-c_{2}^{2}\right)}{2 \sin \left[\frac{1}{3} \sin ^{-1}(z(m))+\frac{\pi}{3}\right]}, \\
& E\left(c_{3}\right)=\frac{3 \sqrt{3} m v^{2}}{2 z(m)}=\frac{3 \sqrt{3} m v^{2}}{2 z(m)}\left(c_{3}^{2}\right)=\frac{\sqrt{3} m c_{3}^{2}}{2 \sin \left[\frac{1}{3} \sin ^{-1}(z(m))\right]} .
\end{aligned}
$$

It is worthwhile to verify the validity of relations (9) with $z$ (m, relative) from (8.2). Here, we concentrate specifically on (9.3). In this connection, assume that $v \approx c$, and as we have that $c_{3}=c$, then also $\beta \approx 1$ with $z(m) \rightarrow z(m$, relative $) \approx$ $3 \sqrt{3} / 2 \gamma \ll 1$. Now, we apply this result to both sides of $(9.3)$ :

$$
\begin{aligned}
z(m) & =z(m, \text { relative }) \approx 3 \sqrt{3} / 2 \gamma \ll 1, v \approx c, c_{3}=c: \\
\frac{3 \sqrt{3} m v^{2}}{2 z(m)} & \approx \gamma m c^{2}, \frac{\sqrt{3} m c_{3}^{2}}{2 \sin \left[\frac{1}{3} \sin ^{-1}(z(m))\right]} \approx \gamma m c^{2} .
\end{aligned}
$$

and because $z(m$, relative $)$ is infinitesimal, both sides, as they should, give $E\left(c_{3}\right) \approx \gamma m c^{2}$, the relativistic energy from (Gricths, D., 1987) and (Eisberg, J. M., 1966), as expected. Other energies $E\left(c_{1}\right)$ and $E\left(c_{2}\right)$, within different context, are discussed later.

In order to obtain the particle limiting velocity solutions in reverse, as functions of the congruent parameter $z$, we first list a series of $z$-identities.

$$
\begin{aligned}
-1 & \leq z \leq 1 \text { Identities: } \\
z & =3 \sin \left[\frac{1}{3}\left(\pi-\sin ^{-1} z(m)\right)\right]-4 \sin ^{3}\left[\frac{1}{3} \pi-\sin ^{-1}(z(m))\right], \\
z & =-3 \sin \left[\frac{1}{3}\left(\pi+\sin ^{-1}(z)\right)\right]+4 \sin ^{3}\left[\frac{1}{3}\left(\pi+\sin ^{-1} z(m)\right)\right], \\
z & =3 \sin \left[\frac{1}{3} \sin ^{-1}(z)\right]-4 \sin ^{3}\left[\frac{1}{3} \sin ^{-1}(z)\right]
\end{aligned}
$$

With little work, the particle limiting velocities in reverse are:

$$
\begin{aligned}
& \frac{v^{2}}{c_{1}^{2}(m)}=\frac{(10.1) z}{3 \sin \left[\frac{1}{3}\left(\pi-\sin ^{-1} z(m)\right)\right]}=1-\frac{4}{3} \sin ^{2}\left[\frac{1}{3}\left(\pi-\sin ^{-1}(z(m))\right]>0\right. \\
& \frac{v^{2}}{c_{2}^{2}(m)}=\frac{(10.2) z}{3 \sin \left[\frac{1}{3}\left(\pi+\sin ^{-1} z(m)\right)\right]}=1-\frac{4}{3} \sin ^{2}\left[\frac{1}{3}\left(\pi+\sin ^{-1}(z(m))\right]<0\right. \\
& \frac{v^{2}}{c_{3}^{2}(m)}=\frac{(10.3) z}{3 \sin \left[\frac{1}{3} \sin ^{-1}(z(m))\right]}=1-\frac{4}{3} \sin ^{2}\left[\frac{1}{3} \sin ^{-1}(z(m))\right]>0
\end{aligned}
$$

Combining relations $(9.1,2,3)$ with appropriate square roots from $(11.1,2,3)$ yield the expressions for equal particle energies, $E\left(c_{i}\right) \Rightarrow E, i=1,2,3$, and the corresponding momenta: 


$$
\begin{aligned}
& E\left(c_{1}\right)=m c_{1}^{2}(m)\left(1-\frac{\vec{v}^{2}}{c_{1}^{2}(m)}\right)^{-\frac{1}{2}} \\
& \vec{p}\left(c_{1}\right)=\frac{E\left(c_{1}\right) \vec{v}}{c_{1}^{2}(m)}=m \vec{v}\left(1-\frac{\vec{v}^{2}}{c_{1}^{2}(m)}\right)^{-\frac{1}{2}} \\
& E\left(c_{2}\right)=-m c_{2}^{2}(m)\left(1-\frac{\vec{v}^{2}}{c_{2}^{2}(m)}\right)^{-\frac{1}{2}}, \\
& \vec{p}\left(c_{2}\right)=\frac{E\left(\left(c_{2}\right) \vec{v}\right.}{\left(-c_{2}^{2}(m)\right)}=m \vec{v}\left(1-\frac{\vec{v}^{2}}{c_{2}^{2}(m)}\right)^{-\frac{1}{2}} \\
& E\left(c_{3}\right)=m c_{3}^{2}(m)\left(1-\frac{\vec{v}^{2}}{c_{3}^{2}(m)}\right)^{-\frac{1}{2}}, \\
& \vec{p}\left(c_{3}\right)=\frac{E\left(c_{3}\right) \vec{v}}{c_{3}^{2}() m}=m \vec{v}\left(1-\frac{\vec{v}^{2}}{c_{3}^{2}(m)}\right)^{-\frac{1}{2}}
\end{aligned}
$$

Although the expressions for $E$ and $\vec{p}$ were derived with $-1 \leq z \leq 1$, these expressions (12) are generally valid as the congruent parameter $z$ does not appear in them explicitly. Now with $(12.1,2,3)$, the mass-shell condition for a particle of mass, $m$, velocity, $v$, and energy, $E\left(c_{i}\right)$ (of the same value $E$ for each $i$ ) with details of primary, secondary and tertiary particle limiting velocities can be written as,

$$
\vec{p}^{2}\left(c_{i}\right) c_{i}^{2}-E^{2}\left(c_{i}\right)+m^{2} c_{i}^{4}=0, i=12,3
$$

At this point we turn to evaluate numerically limiting velocities $c_{1}, c_{2}$, and $c_{3}$ for the muon neutrino, $v(\mu)$, luminal velocity measurement with OPERA detector (Adams, T. et al., 2012) and for the electron, $e$,relativistic luminal velocity measurement using 2010 Crab-Nebula Flare by F. W. Stecker (Stecker, F. W., 2015). In both of these cases, the following general information for respective neutrino,$v(\mu)$, from (Adams, T. et al., 2012) and the electron ,e, from [11] (with $m, v$, $z, E$, etc., associated with either $v(\mu)$ or $(e)$ are assumed to hold :

$$
\begin{aligned}
v & \approx c, m c^{2} \ll E, z \approx \frac{3 \sqrt{3} m c^{2}}{2 E} \ll 1, \\
\sin ^{-1}(z) & \approx z, \sin \left[\frac{1}{3}\left(\sin ^{-1}(z)\right] \approx \frac{1}{3} z\right. \\
\cos \left[\frac{1}{3}\left(\sin ^{-1}(z)\right]\right. & \approx \cos \left(\frac{1}{3} z\right) \approx 1 .
\end{aligned}
$$

With (14), the solutions for limiting velocities from (6), for either $v(\mu)$ or $(e)$ particle,are to a good approximations as follow:

$$
\begin{aligned}
& c_{1}^{2}(m) \approx c^{2}\left[\frac{3 \sqrt{3}}{2 z(m)}-\frac{1}{2}\right] \approx c^{2}\left[\frac{E}{m c^{2}}-\frac{1}{2}\right] \\
& c_{2}^{2}(m) \approx-c^{2}\left[\frac{3 \sqrt{3}}{2 z(m)}+\frac{1}{2}\right] \approx-c^{2}\left[\frac{E}{m c^{2}}+\frac{1}{2}\right] . \\
& c_{3}^{2}(m) \approx c^{2}
\end{aligned}
$$

On specific level, the data plus results for muon neutrino luminal velocity measurements from the OPERA detector (Adams, T. et al., 2012) are: 


$$
\begin{aligned}
& E(v(\mu))=17 \mathrm{GeV}, v(v(\mu)) \approx c \\
& m(v(\mu)) \approx 0.76 \mathrm{eV}, z(v(\mu)) \approx 1.16 \times 10^{-10}: \\
& c_{1}^{2}(v(\mu)) \approx\left(2.24 \times 10^{10}-\frac{1}{2}\right) c^{2}, c_{2}^{2}(v(\mu)) \approx-\left(2.24 \times 10^{10}+\frac{1}{2}\right) c^{2} \\
& c_{3}^{2}(v(\mu)) \approx c^{2} \\
& c_{1}(v(\mu)) \approx 1.5 \times 10^{5} c, c_{2}(v(\mu)) \approx \pm i 1.5 \times 10^{5} c, c_{3}(v(\mu)) \approx c .
\end{aligned}
$$

Similarly, the Stecker's electron data (Stecker, F. W., 2015) with evaluation results are:

$$
\begin{aligned}
& E(e)=5.1 \mathrm{PeV}, v(e) \approx c, m(e) c^{2} \approx 0.51 \mathrm{MeV}, z(e) \approx 2.6 \times 10^{-10}: \\
& c_{1}^{2}(e) \approx\left(10^{10}-\frac{1}{2}\right) c^{2}, c_{2}^{2}(e) \approx-\left(10^{10}+\frac{1}{2}\right) c^{2}, c_{3}^{2}(e) \approx c^{2} ; \\
& c_{1}(e) \approx 10^{5} c, c_{2}(e) \approx \pm i 10^{5} c, c_{3}(e) \approx c .
\end{aligned}
$$

One thing immediately noticeable is that $c_{3} \approx c$ in both the neutrino and electron examples, regardless to large difference between their energies. Similarly, rather "small" difference between $c_{1,2}(e)$ from $c_{1,2}(v(\mu))$ appears not to be affected by large difference between $E(e)$ and $E(v(\mu))$ but rather favored by close numerical values of congruent parameters $z(e) \approx 2.6 \times 10^{-10}$ and $z(v(\mu)) \approx 1.16 \times 10^{-10}$. Actually, with $z(m)$ from $(6.0)$ combined with the fronts of $(6.1,2,3)$ yield three inverted $(6.1,2,3)$ for $z(m)$ which indicates the $z(m)$ constancy with respect to $c_{1}^{2}(m), c_{2}^{2}(m)$ and $c_{3}^{2}(m)$,

$$
\begin{aligned}
& z(m)=\sin \left[\pi-3 \sin ^{-1}\left(\frac{m \sqrt{3} c_{1}^{2}}{2 E}\right)\right], \\
& z(m)=-\sin \left[\pi+3 \sin ^{-1}\left(\frac{m \sqrt{3} c_{2}^{2}}{2 E}\right)\right], \\
& z(m)=\sin \left[3 \sin ^{-1}\left(\frac{m \sqrt{3} c_{3}^{2}}{2 E}\right)\right] .
\end{aligned}
$$

\section{Exact Novel Particle Limiting Velocity Solutions in Qquadratic Complex Forms With Corresponding Real Energies}

As indicated in the Introduction, the particle limiting velocity bicubic equation (4) yields also solutions for novel particles with the discriminant $D \geq 0$ and the squared congruent parameter $z^{2} \geq 1$. This hypothetical novel particle has been discussed in references (Śoln, J., 2019, 2029, 221) and here, we start with the bicubic equation for particle limiting velocities :

$$
\begin{gathered}
\left(\frac{c^{2}}{v^{2}}\right)^{3}-\left(\frac{E}{m v^{2}}\right)^{2}\left(\frac{c^{2}}{v^{2}}\right)+\left(\frac{E}{m v^{2}}\right)^{2}=0, \\
D=\left(\frac{27}{8}\right)^{2} \frac{1}{z^{4}(m)}\left(1-\frac{1}{z^{2}(m)}\right) \geq 0, z^{2} \geq 1, z=\frac{3 \sqrt{3} m v^{2}}{2 E}
\end{gathered}
$$

The original solutions of $(18.1,2)$ are given in (Śoln, J., 2019) as

$$
\begin{aligned}
\frac{c_{1,2}^{2}(m)}{v^{2}}= & \frac{3}{2 z(m)} \csc 2 \tan ^{-1}\left(\tan \left(\frac{1}{2} \sin ^{-1}\left(\frac{1}{z(m)}\right)\right)\right)^{\frac{1}{3}} \\
& \pm i \frac{3 \sqrt{3}}{2 z(m)} \operatorname{ctn} 2 \tan ^{-1}\left(\tan \left(\frac{1}{2} \sin ^{-1}\left(\frac{1}{z(m)}\right)\right)\right)^{\frac{1}{3}}, \\
\frac{c_{3}^{2}(m)}{v^{2}}= & -\frac{3}{z(m)} \csc 2 \tan ^{-1}\left(\tan \left(\frac{1}{2} \sin ^{-1}\left(\frac{1}{z(m)}\right)\right)\right)^{\frac{1}{3}} .
\end{aligned}
$$


As noticed already in (Śoln, J., 2019, 2029, 221), the solutions (19) with $z \geq 1$ practically suggest the introduction of the congruent angle $\alpha(m)$ in terms of the congruent parameter $z(m)$ :

$$
\begin{aligned}
\alpha(m) & =2 \tan ^{-1}\left(\tan \left(\frac{1}{2} \sin ^{-1}\left(\frac{1}{z(m)}\right)\right)\right)^{\frac{1}{3}}, \\
\frac{1}{z(m)} & =\sin \left[2 \tan ^{-1}\left(\tan \left(\frac{\alpha(m)}{2}\right)\right)^{3}\right], \\
z^{2}(m) & \geq 1,0<\alpha(m) \leq \pi / 2 .
\end{aligned}
$$

Then, with the help of (20) the solutions (19), are now in somewhat simplified forms:

$$
\begin{aligned}
c_{1,2}^{2}(m) & =\frac{3[1 \pm i \sqrt{3} \cos (\alpha(m))] v^{2}}{2 z(m) \sin (\alpha(m))}=\operatorname{Re} c_{1,2}^{2}(m)+i \operatorname{Im} c_{1,2}^{2}(m), \\
\operatorname{Re} c_{1,2}^{2}(m) & =\frac{3 v^{2}}{2 z(m) \sin (\alpha(m))}, \operatorname{Im} c_{1,2}^{2}(m)= \pm \frac{3 \sqrt{3} v^{2}}{2 z(m)} c \operatorname{ctn}(\alpha(m)), \\
c_{3}^{2}(m) & =-\frac{3 v^{2}}{z(m) \sin (\alpha(m))} ; \\
\operatorname{Re} c_{1}^{2} & =\operatorname{Re} c_{2}^{2}, \operatorname{Im} c_{1}^{2}=-\operatorname{Im} c_{2}^{2}, c_{3}^{2}=-2 \operatorname{Re} c_{1,2}^{2} .
\end{aligned}
$$

Already a this stage we see the difference between ordinary particles and novel particles. Here, by and large, we have that limiting velocities $c_{1}, c_{2}$ and $c_{3}$,associated respectively with primary, secondary and tertiary novel particles, are generally complex quantities. Furthermore, the global interrelations between the novel particle limiting velocities is easily established from (21).

Next, we wish to show that the congruent angle $\alpha$ and parameter $z$, in addition to (20), satisfy more relations which will further reaffirm that $z(m)$ and $\alpha(m)$ are real. To do so, from relations $(18.2)$ one first notices the relation, $E / m v^{2}=3 \sqrt{3} / 2 z$, which is substituted into bicubic equation from (18.1) to yield with notations from (21) relations with real and imaginary parts:

$$
\begin{aligned}
\frac{E}{m v^{2}} & =\frac{3 \sqrt{3}}{2 z}, \\
\operatorname{Re}, \operatorname{Im}\left[\left(\frac{c_{i}^{2}}{v^{2}}\right)^{3}-\frac{27}{4 z^{2}(m)}\left(\frac{c_{i}^{2}}{v^{2}}\right)+\frac{27}{4 z^{2}(m)}\right] & =0, i=12,3
\end{aligned}
$$

Since the solutions for $c_{1,2,3}^{2}(m)$ are known from (21), relations (22) are basically two equations for $z(m)$ with $\alpha(m)$. Utilizing solutions (21), we start with the real part containing $c_{1,2}^{2}$ to yield relationship between $z(m)$ and $\alpha(m)$.

$$
\begin{aligned}
\operatorname{Re}\left[\left(\frac{c_{1,2}^{2}}{v^{2}}\right)^{3}-\frac{27}{4 z^{2}(m)}\left(\frac{c_{1,2}^{2}}{v^{2}}\right)+\frac{27}{4 z^{2}(m)}\right] & =0: 1-9 \cos (\alpha(m)) \\
-3 \sin ^{2}(\alpha(m))+2 z(m) \sin ^{3}(\alpha(m)) & =0 \\
z(m) & =\frac{1+3 \cos ^{2}(\alpha(m))}{\sin ^{3}(\alpha(m))} .
\end{aligned}
$$

The resulting $(z(m)$ is quite different in form from the one in (20) but numerically the same, as we will be able to verify shortly. Similarly addressing the imaginary part of $c_{1,2}^{2}$ in (22), which with the help of (21) yields simple result that $\alpha(m)$ is real.

$$
\begin{aligned}
& \operatorname{Im}\left[\left(\frac{c_{1,2}^{2}}{v^{2}}\right)^{3}-\frac{27}{4 z^{2}(m)}\left(\frac{c_{1,2}^{2}}{v^{2}}\right)\right]=0: \\
& \mp \cos ^{2}(\alpha(m)) \pm 1 \mp \sin ^{2}(\alpha(m))=0 .
\end{aligned}
$$


Finally we come to $c_{3}^{2}$ in (22) which with solution in (21) yields the same result for $z(m)$ as in (23)

$$
\begin{aligned}
\left(\frac{c_{3}^{2}}{v^{2}}\right)^{3}-\frac{27}{4 z^{2}(m)}\left(\frac{c_{3}^{2}}{v^{2}}\right)+\frac{27}{4 z^{2}(m)} & =0: \\
-\frac{1}{\sin ^{3}(\alpha(m))}+\frac{3}{4 \sin (\alpha(m))}+\frac{z(m)}{4} & =0, \\
z(m) & =\frac{4-3 \sin ^{2}(\alpha(m))}{\sin ^{3}(\alpha(m))}
\end{aligned}
$$

The advantage of having (23) and (25) expressions for $z(m)$ is that now congruent parameter $z(m)$ can be eliminated in favor of just congruent angle $\alpha(m)$ in most of the expressions, if so desired. This can be summarized by also expressing the discriminant and the energy in just the terms of $\alpha(m)$ :

$$
\begin{aligned}
z(m) & =\frac{1+3 \cos ^{2}(\alpha(m))}{\sin ^{3}(\alpha(m))} \geq 1,0<\alpha(m) \leq \frac{\pi}{2}, \\
D(m) & =\left(\frac{27}{8}\right)^{2} \frac{\sin ^{12}(\alpha(m))}{\left(1+3 \cos ^{2}(\alpha(m))^{4}\right.}\left(1-\frac{\sin ^{6}(\alpha(m))}{\left(1+3 \cos ^{2}(\alpha(m))^{2}\right.}\right), \\
E(m) & =\frac{3 \sqrt{3} \sin ^{3}(\alpha(m))}{\left(1+3 \cos ^{2}(\alpha(m))\right.} \frac{m v^{2}}{2}, 0<\alpha(m) \leq \frac{\pi}{2} .
\end{aligned}
$$

In what follows, the primary, secondary and tertiary quadratic and, yet to be discussed linear, novel particle limiting velocities can be presented with just the congruent angle e $\alpha(m)$, when more convenient. This particularly so if some novel particles may assume also superluminal velocity values. To be prepared to those possbilities, consistent with the relations (20)-(26), in Table 2 the calculated values of $\alpha(m)$ from $z(m)$ are presented.

Table 2. Congruent parameters $\alpha(m)$ and $z(m)$ values to third decimal point approximation

$$
\left(\begin{array}{cccccccc}
\alpha(m): & \frac{\pi}{2} & \frac{\pi}{2.25} & \frac{\pi}{2.3} & \frac{\pi}{2.5} & \frac{\pi}{2.75} & \frac{\pi}{3} & \frac{\pi}{3.25} \\
\frac{1}{z(m)}: & 1 & 0.876 & 0.835 & 0.669 & 0.496 & 0.371 & 0.283 \\
z(m): & 1 & 1.142 & 1.198 & 1.495 & 2.016 & 2.694 & 3.531
\end{array}\right)
$$

Next, we continue in new directions,by conecting the energies of novel particles to their velocity squares $v^{2}$, their squares of primary, secondary $\operatorname{Re} c_{1,2}^{2}(\alpha(m))$, primary, secondary $\operatorname{Im} c_{1,2}^{2}(\alpha(m))$, the whole primary, secondary $c_{1,2}^{2}(\alpha(m))$ and to tertiary $c_{3}^{2}(\alpha(m))$, respectively for each of the primary,secondary and tertiary novel particles. As relation (5) indicates, to evaluate the energy of either ordinary or novel particle the particle's ratio $v^{2} / z(m)$ needs to be known. To this effect, specifically from $(21)$ with $v^{2} / z(m)$ we derive the relations between squares of particle usual and limiting velocities, necessary in derivation of novel particle energies. To this end, similarly as for the ordinary articles $(9.1,2,3)$, here also the applications of $v^{2} / z(m)$ to the real and imaginary portions, separate or together, of limiting velocity solutions (21), yield the following expressions:

$$
\begin{aligned}
c_{1,2}^{2}: & \frac{v^{2}}{z(m)}=\frac{2 \sin (\alpha(m)) c_{1,2}^{2}[1 \mp i \sqrt{3} \cos (\alpha(m))]}{3\left[1+3 \cos ^{2}(\alpha(m))\right]} \\
= & \frac{2 \sin (\alpha(m))}{3\left[1+3 \cos ^{2}(\alpha(m))\right]}\left[\operatorname{Re} c_{1,2}^{2} \pm \sqrt{3} \cos (\alpha(m)) \operatorname{Im} c_{1,2}^{2}\right. \\
& \left.+i\left(\operatorname{Im} c_{1,2}^{2} \mp \sqrt{3} \cos (\alpha(m)) \operatorname{Re} c_{1,2}^{2}\right)\right] .
\end{aligned}
$$




$$
\begin{aligned}
\operatorname{Re} \text { ality }: & i\left(\operatorname{Im} c_{1,2}^{2} \mp \sqrt{3} \cos (\alpha(m)) \operatorname{Re} c_{1,2}^{2}\right)=0 \\
c_{1,2}^{2}: & \frac{v^{2}}{z(m)}=\frac{2 \sin (\alpha(m))}{3\left[1+3 \cos ^{2}(\alpha(m))\right]}\left[\operatorname{Re} c_{1,2}^{2}\right. \\
& \left. \pm \sqrt{3} \cos (\alpha(m)) \operatorname{Im} c_{1,2}^{2}\right], \\
\operatorname{Re} c_{1,2}^{2}: & \frac{v^{2}}{z(m)}=\frac{2}{3} \sin (\alpha(m)) \operatorname{Re} c_{1,2}^{2}, \\
\operatorname{Im} c_{1,2}^{2}: & \frac{v^{2}}{z(m)}= \pm \frac{2}{3 \sqrt{3}} \tan (\alpha(m)) \operatorname{Im} c_{1,2}^{2}, \\
c_{3}^{2}: & \frac{v^{2}}{z(m)}=-\frac{\sin (\alpha(m))}{3} c_{3}^{2} .
\end{aligned}
$$

Let us point out that the reality condition (27. 2) is already contained in relations (21). Here, with (27.2), together with real and imaginary parts from (27.1), (27.2) one transforms (27.1) into (27.3)),(27.4) and (27.5) while (27.6) is due to the inversion from (21). With (27.3)-(27.6) we have complete quadratic limiting velocity presentations for evaluating respective primary, secondary and tertiary novel particle energies. To this effect, it is worthwhile to see how the values of the congruent angle $\alpha(m)$ from Table 1., according to the quadratic limiting velocity solutions (21) may affect such calculations,

$$
\begin{aligned}
z(m) & =1, \alpha(m)=\frac{\pi}{2} \\
& : \quad c_{1,2}^{2}=\operatorname{Re} c_{1,2}^{2}=\frac{3}{2} v^{2}, \operatorname{Im} c_{1,2}^{2}=0, c_{3}^{2}=-3 v^{2}, \\
z(m)= & 1.495, \alpha(m)=\frac{\pi}{2.5} \\
: & c_{1,2}^{2}=1.055 v^{2} \pm i 0.565 v^{2}, c_{3}^{2}=-2.1 v^{2} .
\end{aligned}
$$

The novel particle energies labeled by specific limiting velocities, according to (5), together with properties (27. 1-8), will follow. We start with the exemplary general expression:

$$
E\left(c_{1,2,3}^{2}(m)\right)=\frac{3 \sqrt{3} m}{2} \cdot \frac{v^{2}}{z(m)}\left(c_{1,2,3}^{2}(m)\right)
$$

Next, in the fashion of (5), we deduce from (27.1-5) the following dark matter particle energies for the primary $\left(c_{1}^{2}\right)$ and secondary $\left(c_{2}^{2}\right)$ dark matter particles:

$$
\begin{aligned}
E\left(c_{1,2}^{2}(m)\right)= & \frac{3 \sqrt{3} m}{2} \cdot \frac{v^{2}}{z(m)}\left(c_{1,2}^{2}(m)\right) \\
= & \sqrt{3} m \sin (\alpha(m)) c_{1,2}^{2}(m) \frac{[1 \mp i \sqrt{3} \cos (\alpha(m))]}{\left[1+3 \cos ^{2}(\alpha(m))\right]} \\
= & \frac{\sqrt{3} m 3 v^{2}[1 \pm i \sqrt{3} \cos (\alpha(m))][1 \mp i \sqrt{3} \cos (\alpha(m))]}{2 z(m)\left[1+3 \cos ^{2}(\alpha(m))\right]} \\
& \left(=\frac{3 \sqrt{3} m v^{2}}{2 z(m)}\right) .
\end{aligned}
$$

In this evaluation, the reality condition is automatically taken into account without specifying it in the course of evaluation with the solutions of limiting velocities (27.1-5)). This is so, since from the limiting velocity solutions (21) for the primary $\left(c_{1}\right)$ and secondary $\left(c_{2}\right)$ particles, one has implicitly that $\operatorname{Im} c_{1,2}^{2}= \pm \sqrt{3} \cos (\alpha(m)) \operatorname{Re} c_{1,2}^{2}$. Similarly we can evaluate the energy of the tertiary $\left(c_{3}\right)$ particle from (27.6),

$$
E\left(c_{3}^{2}(m)\right)=\frac{3 \sqrt{3} m}{2} \cdot \frac{v^{2}}{z(m)}\left(c_{3}^{2}\right)=-\frac{\sqrt{3} m \sin (\alpha(m)) c_{3}^{2}}{2}\left(=\frac{3 \sqrt{3} m v^{2}}{2 z(m)}\right)
$$


All three of these, numerically equal, energies are real despite the fact that (primary, secondary) novel particle's squares of limiting velocities, $c_{1,2}^{2}$, are complex. Just knowing $\operatorname{Re} c_{1,2}^{2}$ or $\operatorname{Im} c_{1,2}^{2}$ one can still find out the corresponding energies of which each, as we shall see, equals the energy appearing in $(28.1,2)$. Specifically, for $\operatorname{Re} c_{1,2}^{2}$ and $\operatorname{Im} c_{1,2}^{2}$ from respectively (27. 4) and (27. 5) we write

$$
\begin{aligned}
E\left(\operatorname{Re} c_{1,2}^{2}(m)\right) & =\frac{3 \sqrt{3} m}{2} \cdot \frac{v^{2}}{z(m)}\left(\operatorname{Re} c_{1,2}^{2}\right) \\
& =\sqrt{3} m \sin (\alpha(m)) \operatorname{Re} c_{1,2}^{2}\left(=\frac{3 \sqrt{3} m v^{2}}{2 z(m)}\right), \\
E\left(\operatorname{Im} c_{1,2}^{2}(m)\right) & =\frac{3 \sqrt{3} m}{2} \cdot \frac{v^{2}}{z(m)}\left(\operatorname{Im} c_{1,2}^{2}\right) \\
& = \pm m \tan \alpha(m) \operatorname{Im} c_{1,2}^{2}\left(=\frac{3 \sqrt{3} m v^{2}}{2 z(m)}\right) .
\end{aligned}
$$

The common value of energy $3 \sqrt{3} m v^{2} / 2 z(m)=3 \sqrt{3} m v^{2} \sin ^{3}(\alpha(m)) / 2\left(1+3 \cos ^{2}(\alpha(m))\right.$ in (5. 1-5) follows from solutions for (primary, secondary) $c_{1,2}^{2}(m), \operatorname{Re} c_{1,2}^{2}(m)$ and $\operatorname{Im} c_{1,2}^{2}(m)$ in $(2.1,2)$ or $(5.4,5)$ and for (tertiary) $c_{3}^{2}(m)(2.3)$ limiting velocity squares. To change the energy one changes the value of $z(m)$ or equivalently of $\alpha(m)$. The easiest way to exhibit that is to take specific congruent parameters with unspecified novel particle mass and velocity and inserting them into (5. $1,2)$ or $(5.4,5)$ :

$$
\begin{aligned}
\alpha(m) & =\frac{\pi}{2.5}, z(m)=1.495 \\
& : \operatorname{Re} c_{1,2}^{2}=1.055 v^{2}, \operatorname{Im} c_{1,2}^{2}= \pm 0.565 v^{2}, c_{3}^{2}=-2.1 v^{2}, \\
E\left(c_{1,2}^{2}(m)\right) & \simeq 1.738 m v^{2}, E\left(\operatorname{Re} c_{1,2}^{2}(m)\right) \simeq 1.738 m v^{2}, \\
E\left(\operatorname{Im} c_{1,2}^{2}(m)\right) & \simeq 1.739 m v^{2}, E\left(c_{3}^{2}(m)\right) \simeq 1.729 m v^{2} .
\end{aligned}
$$

One may see the energy as a sacred quantity, as all these energy expressions, with fixed $z(m)$ and $\alpha(m)$, give the same value even from $\operatorname{Im} c_{1}^{2}(m)$ ) (energy changes only if $z(m)$ or equivalently $\alpha(m)$ changes). This particularly so, as for any energy, the zero sum rule for squares of limiting velocities holds for both real and imaginary ones.

$$
\left.\left.\left.\left.\operatorname{Re} c_{1}^{2}(m)\right)+\operatorname{Re} c_{2}^{2}(m)\right)+c_{3}^{2}=0, \quad \operatorname{Im} c_{1}^{2}(m)\right)+\operatorname{Im} c_{2}^{2}(m)\right)=0 .
$$

As long as the congruent parameters satisfy $z(m) \neq 1$ and $\alpha(m) \neq \pi / 2$, novel particle energies and momenta appear not to be expressible in the Lorentzian like forms as in (Śoln, J., 2019). However, as also in (Śoln, J., 2019), they are expressible in more general usual forms. All the numerically equal energy expressions, including $E\left(\operatorname{Im} c_{1,2}^{2}(m)\right)$, are real, indicating energy as fundamental quantity in physics. The particle momentum is more a quantity of convenience, as its real value is associated with $\operatorname{Re} c_{1}^{2}(m)$ or $c_{3}^{2}$ but not also with $\operatorname{Im} c_{1}^{2}(m)$.

The novel particle momenta are defined with their energies, preferably in such a way, as to be real in values. With that in mind, we write down the particle momenta for primary, secondary and tertiary novel particles,

$$
\begin{aligned}
\vec{p}\left(c_{1,2}^{2}\right) & =\vec{p}\left(\operatorname{Re} c_{1,2}^{2}\right)=\frac{E\left(c_{1,2}^{2}(m)\right) \vec{v}}{\operatorname{Re} c_{1,2}^{2}}=\sqrt{3} m \vec{v} \sin (\alpha(m)), \\
\vec{p}\left(c_{3}^{2}\right) & =\frac{E\left(c_{3}^{2}(m)\right) \vec{v}}{\left(-c_{3}^{2}(m)\right)}=\frac{\sqrt{3}}{2} m \vec{v} \sin (\alpha(m)), 0<\alpha(m) \leq \frac{\pi}{2} .
\end{aligned}
$$

For primary,secondary novel particles with $c_{1,2}$ limiting velocities, in the definitions we use (primary, secondary) $\operatorname{Re} c_{1,2}^{2}(m)$ with $E\left(\operatorname{Re} c_{1,2}^{2}(m)\right.$ ) (numerically equal to $E\left(c_{1,2}^{2}(m)\right)$ ) to define the equal value momenta of which each in form is very similar to tertiary particle momentum but double in value. What we have here is basically the same energy particle but with with variety of physical attributes. 


\section{Linear Forms of Complex Limiting Velocity Solutions for Novel Particles With Real Energies}

The linearization of particle limiting velocities from (21) will be done in analog of linearizing of these quadratic bionomical equations yielding, with the definition of $r$, by trial the linearized $d(+,-)$ as indicated (Rade, L., \& Westergren, B., 1999, p. 65):

$$
\begin{aligned}
d^{2}(+,-) & =a+i( \pm b), r=\sqrt{a^{2}+( \pm b)^{2}}=\sqrt{a^{2}+b^{2}} \\
d(+) & = \pm\left[\sqrt{\frac{r+a}{2}}+i \sqrt{\frac{r-a}{2}}\right] \\
d(-) & = \pm\left[\sqrt{\frac{r+a}{2}}-i \sqrt{\frac{r-a}{2}}\right]
\end{aligned}
$$

whose verification backward is easy to carry out. The linearizations of $c_{1}^{2}(m)$ and $c_{2}^{2}(m)$ from $(21)$ will be done respectively with substitutions

$$
\begin{aligned}
d(+) & =c_{1}(m), d(-)=c_{2}(m) \\
a & =\frac{3 v^{2}}{2 z(m) \sin (\alpha(m))}, b=\frac{3 \sqrt{3} \operatorname{ctn}\left((\alpha(m)) v^{2}\right.}{2 z(m)}, \\
r^{2} & =\left(\frac{3 v^{2}}{2(z(m) \sin (\alpha(m))}\right)^{2}\left(1+3 \cos ^{2}(\alpha(m))\right)
\end{aligned}
$$

With proper substitutions from $(30.2,3,4)$ into $(30.1)$ one arrives at linear expressions of primary, secondary and also, directly from (21), tertiary limiting velocities, $c_{1}, c_{2}$ and $c_{3}$,

$$
\begin{aligned}
c_{1,2}(m)= & ( \pm v)\left(\frac{3}{4 z(m) \sin (\alpha(m))}\right)^{1 / 2} \times \\
& \times\left[\left(1+\sqrt{\left(1+3 \cos ^{2}(\alpha(m))\right.}\right)^{1 / 2}+i,-i\left(\sqrt{\left(1+3 \cos ^{2}(\alpha(m))\right.}-1\right)^{1 / 2}\right], \\
c_{3}(m)= & i( \pm v) \sqrt{\frac{3}{z(m) \sin (\alpha(m))}}, 0<\alpha(m) \leq \frac{\pi}{2}
\end{aligned}
$$

These, when squared become exactly $(21)$ and obey $D(m) \geq 0, z^{2}(m) \geq 1$,indicating that they describe the novel particles. One notices preservations of complexities for $c_{1}(m)$ and $c_{2}(m), c_{1,2}^{*}(m)=c_{2,1}(m)$, but not the reality for $c_{3}(m), c_{3}^{*}(m)$ $=-c_{3}(m)$, The simple sample values of congruent quantities $\alpha(m)$ and $z(m)$ from $(27.7)$ and $(27.8), \alpha(m)=\pi / 2, \pi / 2.5$ with respective $z(m)=1,1.495$, from numerical evaluations of quadratic limiting velocities $c_{1,2}^{2}(m)$, are also used here when evaluating linear limiting velocities $c_{1,2}(m)$ and $c_{3}(m)$ from $(30.5,6)$,

$$
\begin{aligned}
z(m) & =1, \alpha(m)=\frac{\pi}{2} \\
& : \quad c_{1,2}=\operatorname{Re} c_{1,2}=( \pm v) \sqrt{\frac{3}{2}}, \operatorname{Im} c_{1,2}=0, c_{3}=i( \pm v) \sqrt{3} \\
z(m)= & 1.495, \alpha(m)=\frac{\pi}{2.5} \\
& : \quad c_{1,2}=( \pm v)(1.061+i,-i 0.266), c_{3}=i( \pm v) 1.45 .
\end{aligned}
$$

One notices the numerical consistencies between respective relations of (30.7), (30.8) with (27.7),(27.8), by squaring (30.7) and (30.8) and taking inti account that in general,

$$
\begin{aligned}
& \operatorname{Re} c_{1,2}^{2}=\left(\operatorname{Re} c_{1,2}\right)^{2}-\left(\operatorname{Im} c_{1,2}\right)^{2} \\
& \operatorname{Im} c_{1,2}^{2}=2 \operatorname{Re} c_{1,2} \operatorname{Im} c_{1,2}, c_{3}^{2}=\left(c_{3}\right)^{2}
\end{aligned}
$$


The relations (30.9) and (30.10), although simple, in fact are important to take into account, when operating with linear form limiting velocities $c_{1,2}$, but evaluating physical quantities depending on either $c_{1,2}^{2}$ or $\operatorname{Re} c_{1,2}^{2}$ and even on $\operatorname{Im}$ $c_{1,2}^{2}$. Relation (30.9) is also good example of how, for novel particles, even imaginary limiting velocity expressions can contribute to the real ones and to the real results.

\section{Just Congruent Angle $\alpha(\mathrm{m})$ Description of Novel Particle Limiting Velocities With Real Energies}

Working in Section 3 on solutions of novel particle limiting velocities (21), the necessity of the congruent angle $\alpha(m)$ related to the congruent parameter $z(m)$, got firmly established. Their rather strong nonlinear relationships are already given in relations (20). However, studying real and imaginary parts of particle limiting velocity expressions, a relatively simpler nonlinear relationship between $z((m)$ and $\alpha(m)$ got established, as shown in (26). The big advantage of this expression for the novel particle energy is in the fact that it does not involve directly $\left.\operatorname{Re} c_{1,2}^{2}(m)\right)$ and $\operatorname{Im} c_{1,2}^{2}(m)$ ), which was already carried out in relations (28) to (28.6). Some practical aspects will be mentioned briefly also in the Section V.

Here, however using the new expression in (26) for $z(\alpha(m))$ in terms of $\alpha(m)$ we turn to the novel particle limiting velocities, in both, quadratic and linear forms as expressions in terms of $\alpha(m)$. First,for limiting velocities in quadratic forms,

$$
\begin{aligned}
& \text { (21) }: \quad c_{1,2}^{2}(m)=\frac{3[1 \pm i \sqrt{3} \cos (\alpha(m))] v^{2} \sin ^{2}(\alpha(m))}{2\left(1+3 \cos ^{2}(\alpha(m))\right)} \\
&= \operatorname{Re} c_{1,2}^{2}(m)+i \operatorname{Im} c_{1,2}^{2}(m), 0<\alpha(m) \leq \frac{\pi}{2}, \\
&(21) \quad: \quad \operatorname{Re} c_{1,2}^{2}(m)=\frac{3 v^{2} \sin ^{2}(\alpha(m))}{2\left(1+3 \cos ^{2}(\alpha(m))\right)}, 0<\alpha(m) \leq \frac{\pi}{2}, \\
& \operatorname{Im} c_{1,2}^{2}(m) \quad=\quad \frac{3 \sqrt{3} v^{2} \sin ^{2}(\alpha(m)) \cos (\alpha(m))}{2\left(1+3 \cos ^{2}(\alpha(m))\right)}, 0<\alpha(m) \leq \frac{\pi}{2}, \\
&(21): c_{3}^{2}(m)==-\frac{3 v^{2} \sin ^{2}(\alpha(m))}{\left(1+3 \cos ^{2}(\alpha(m))\right) .}, 0<\alpha(m) \leq \frac{\pi}{2} .
\end{aligned}
$$

Second, for limiting velocities in linear forms expressed in terms of $\alpha(m)$,

$$
\begin{aligned}
(30.5,6):= & c_{1,2}(m)=( \pm v) \frac{\sqrt{3} \sin (\alpha(m))}{2} \times \\
& {\left[\left(\frac{1+\sqrt{1+3 \cos ^{2}(\alpha(m))}}{1+3 \cos ^{2}(\alpha(m))}\right)^{1 / 2} \pm i\left(\frac{\sqrt{1+3 \cos ^{2}(\alpha(m))}-1}{1+3 \cos ^{2}(\alpha(m))}\right)^{1 / 2}\right] } \\
c_{3}(m)= & i( \pm v) \sqrt{3} \frac{\sin (\alpha(m))}{\sqrt{1+3 \cos ^{2}(\alpha(m)}}, 0<\alpha(m) \leq \frac{\pi}{2} .
\end{aligned}
$$

With $z(m)$ from (23) the energy expression from (5) becomes in form the one from from (26), showing that the congruent angle $\alpha(m)$ is an evolutionary parameter whose changing value from $\pi / 2$ to 0 changes the energy $E$ from $3 \sqrt{3}\left(m v^{2} / 2\right)$ to 0 .All these novel particle limiting velocity solutions obey $D(m) \geq 0, z^{2}(m) \geq 1$ from (5).

The new expression for the congruent parameter $z(\alpha(m))$ in (23) or (26) when applied to(20) yield two amusing (but correct) self-identities, for $0<\alpha(m) \leq \frac{\pi}{2}$ :

$$
\begin{gathered}
\alpha(m)==2 \tan ^{-1}\left(\tan \left(\frac{1}{2} \sin ^{-1}\left(\frac{\sin ^{3}(\alpha(m))}{\left(1+3 \cos ^{2}(\alpha(m))\right)}\right)\right)\right)^{\frac{1}{3}}, \\
\frac{\sin ^{3}(\alpha(m))}{\left(1+3 \cos ^{2}(\alpha(m))\right)}=\sin \left[2 \tan ^{-1}\left(\tan \left(\frac{\alpha(m)}{2}\right)\right)^{3}\right]
\end{gathered}
$$


which are the results of more than one representation of the congruent parameter $z(m)$ in terms of the congruent angle $\alpha(m)$.

\section{Discussion With Example of the Spontaneous Transition From Ordinary to Novel Particle and Conclusion}

With comparison of Section 2 with Section 3, one realizes that the congruent parameter $z$ serves as a particle evolutionary parameter. For ordinary particles with values $z \leq 1$ while for novel particles with values $z \geq 1$, and $z=1$ serving as dividing point. At $z=1$ also the congruent angle $\alpha=\pi / 2$ occur with values extending only for $z \geq 1$ with $\alpha \leq \pi / 2$.

The ordinary particles, among which are well known particles such as electrons, neutrinos and the like, all have three limiting velocities $c_{1}, c_{2}$ and $c_{3}$ of which $c_{1}$ and $c_{3}$ are real while $c_{2}$ is imaginary. In fact, from muon neutrino luminal velocity measurement with OPERA detector (Adams, T. et al., 2012) and the electron relativistic luminal velocity measurement using Crab-Nebula Flare by Stecker (Stecker, F. W., 2015), one deduces that $c_{3} \approx c$, the velocity of light. However, the real limiting velocity $c_{1}$ and imaginary $c_{2}$, both with very large absolute values, numerically are different in (Adams, T. et al., 2012) \& (Stecker, F. W., 2015). And, as relations $(9.1,2,3),(12.1,2,3),(14-17)$ indicate, for each trio solutions $\left(c_{1}, c_{2}\right.$ and $c_{3}$ ) the energy $E$ is numerically the same.

The strong difference of novel particles from the ordinary particles is reflected particularly in remarkable differences between their limiting velocity expressions $(6.0,1,2,3)$ for ordinary particles with $z \leq 1$, and expressions (19),(20) and (21) for novel particles with $z \geq 1, \alpha \leq \pi / 2$. However, on general grounds, the global energy expression (5), for ordinary as well as for novel particles, regardless on the complexities of limiting velocities is $E=3 \sqrt{3} m v^{2} / 2 z, z \leq 1, z \geq 1$.

One notices interesting things happening to novel particles once the congruent parameter $z(m) \geq 1$ and $\alpha \leq \pi / 2$. As one sees, the nonlinearly connected dimensionless congruent parameter $z(m)$ and dimensionless congruent angle $\alpha$ are essential in evaluating not only all forms of particle energies but also of linear particle momenta. For one thing, the Lorentzian like form is not favored by either the novel particle energy or the momentum as seen respectively in each of (28.1-.7) and (29.1-.3). Like for the ordinary particles, the amazing thing is again, that different forms of novel particle complex limiting velocity-squares (primary,secondary) $c_{1,2}^{2}(m)$, (primary, secondary) $\operatorname{Re} c_{1,2}^{2}(m)$ and (primary, secondary) $\operatorname{Im} c_{1,2}^{2}(m)(21)$ separately yield the same value novel particle energy $E\left(c_{1,2}^{2}(m)\right)$,while the real (tertiary) $c_{3}^{2}(m)(21)$ yields $E\left(c_{3}^{2}(m)\right.$ (numerically equal to $E\left(c_{1,2}^{2}(m)\right)$ ). Similarly, one has the expressions for novel particle momenta, $\vec{p}\left(c_{1,2}^{2}\right)=$ $\vec{p}\left(\operatorname{Re} c_{1,2}^{2}\right)$ from $E\left(c_{1,2}^{2}(m)\right)$ together with (primary, secondary) $\operatorname{Re} c_{1,2}^{2}(m)$; while tertiary novel particle momentum $\vec{p}\left(c_{3}^{2}\right)$ follows in a usual way from limiting velocity-square $c_{3}^{2}(m)$ and the energy $E\left(c_{3}^{2}(m)\right)$. Unlike the ordinary particles, in Sect. 4. the novel particles allow also straightforward evaluation of limiting velocities in linear forms, while in Sect. $\mathbf{5}$. even in terms of just congruent angle $\alpha(m)$.

Now, K. C. Y. Ng et al. (Ng, Kenney C. Y., et al., 2019), from NuSTAR M31 observations (Ng, Kenney C. Y., et al., 2019), have suggested a sterile neutrino $\chi$ of mass energy $m_{\chi} \geq 12 \mathrm{keV} / \mathrm{c}^{2}$, as a "dark matter" particle, that can decay radioactively into monoenergetic photon $\gamma$ plus active neutrino, $v, \chi \longrightarrow \gamma+v$.

Here, we choose the same mass energy of $m_{\chi} \geq 12 \mathrm{keV} / \mathrm{c}^{2}$ as in (Ng, Kenney C. Y., et al., 2019) to model the new sterile neutrino first as as an ordinary particle with $z \leq 1$ spontaneously transiting via $z(m)=1$ into the novel sterile neutrino with $z \geq 1, \alpha \leq \pi / 2$. On a specific level, consistent with the ordinary sterile neutrino quadratic limiting velocity solutions in $(6.0,1,2,3)$ at $z \leq 1 ; z=1$, the ordinary sterile neutrino of $m_{\chi}=12 \mathrm{keV} / \mathrm{c}^{2}$ has quadratic limiting velocity solutions:

As ordinary particle :

$$
z \leq 1 ; z=1: c_{1}^{2}=1.5 v^{2}, c_{2}^{2}=-3 v^{2}, c_{3}^{2}=1.5 v^{2} .
$$

On the other hand from the novel sterile neutrino quadratic limiting velocity solutions in $(18,19,20,21)$ at $z \geq 1 \longmapsto 1$, the novel sterile neutrino of $m_{\chi}=12 \mathrm{keV} / \mathrm{c}^{2}$ yields the quadratic limiting velocity solutions:

As novel particle :

$$
\begin{aligned}
& z \geq 1 ; z=1: \operatorname{Re} c_{1,2}^{2}=1.5 v^{2}, \operatorname{Im} c_{1,2}^{2}=0, c_{3}^{2}=-3 v^{2}, \\
& z=1.198: \operatorname{Re} c_{1,2}^{2}=1.28 v^{2}, \operatorname{Im} c_{1,2}^{2}= \pm 0.45 v^{2}, c_{3}^{2}=-2.55 v^{2}, \\
& z=1.495: \operatorname{Re} c_{1,2}^{2}=1.055 v^{2}, \operatorname{Im} c_{1,2}^{2}= \pm 0.565 v^{2}, c_{3}^{2}=-2.1 v^{2} .
\end{aligned}
$$

Here we assumed that an ordinary sterile neutrino from $z \leq 1$ spontaneously changed to a novel sterile neutrino with $z \geq 1$. The transition is actually quite straightforward as there are no imaginary components of limiting velocities involved at $z=1$ which, however, will start occurring at $z>1$. 
From Table 1, one notices empirically, that "relativistic" particles with relative velocity restriction $0<\beta<1$ empirically restricts also the congruent parameter to $0<z<1$. By association, one may assume that the novel particles with $z>1$ are likely candidates of the so called "dark matter" particles that are still invisible.

At this point, it is worthwhile (with the help from Table 2, relating congruent parameter $z$ with congruent angle $\alpha$ to express for novel particle, the quadratic limiting velocities(31.1,2,3) as well as linear limiting velocities (31.4) just in terms of the congruent angle $\alpha$ with few examples for $m_{\chi} \simeq 12 \mathrm{keV} / \mathrm{c}^{2}$ :

$$
\begin{aligned}
m_{\chi} & \simeq 12 \mathrm{keV} / \mathrm{c}^{2} \\
\alpha\left(m_{\chi}\right) & =\pi / 2.5: \operatorname{Re} c_{1,2}^{2}=1.055 v^{2}, \operatorname{Im} c_{1,2}^{2}= \pm 0.565 v^{2}, c_{3}^{2}=-2.110 v^{2}, \\
\operatorname{Re} c_{1,2} & =1.061 v, \operatorname{Im} c_{1,2}= \pm 0.266 v, c_{3}=i 1.4525 v ; \\
\alpha\left(m_{\chi}\right) & =\pi / 2.3: \operatorname{Re} c_{1,2}^{2}=1.279 v^{2}, \operatorname{Im} c_{1,2}^{2}= \pm 0.451 v^{2}, c_{3}^{2}=-2.551 v^{2}, \\
\operatorname{Re} c_{1,2} & =1.148 v, \operatorname{Im} c_{1,2}= \pm 0.194 v, c_{3}=i 1.597 v ; \\
\alpha\left(m_{\chi}\right) & =\pi / 2: \operatorname{Re} c_{1,2}^{2}=1.5 v^{2}, \operatorname{Im} c_{1,2}^{2}= \pm 0 v^{2}, c_{3}^{2}=-3 v^{2}, \\
\operatorname{Re} c_{1,2} & =1.225 v, \operatorname{Im} c_{1,2}=0 v, c_{3}=i 1.732 v .
\end{aligned}
$$

As relations $28.1,2, \ldots, 7))$ to $(29.1,2,3)$ indicate that also quadratic form limiting velocities can be used directly to evaluate physical quantities. These are easy to obtain from the linear ones from $(30.9,10)$ yielding now from $(31.1,2,3)$ the necessary quadratic forms:

Next, the evaluation of novel particle energies is in order. The novel particle energy for any limiting velocity form, but of desired congruent parameter $z\left(m_{\chi}\right)$ with corresponding congruent angle $\alpha\left(m_{\chi}\right)$, one can evaluate according to (28) to (28.7), in which both both $z\left(m_{\chi}\right)$ and $\alpha\left(m_{\chi}\right)$,are involved. Equivalently the same energy is evaluated directly from (26) involving just $\alpha\left(m_{x}\right)$,which, being the evolutionary parameter, determines the energy in parallels with limiting velocities.

$$
\begin{aligned}
m_{\chi} & \simeq 12 \mathrm{keV} / \mathrm{c}^{2} \\
\alpha\left(m_{\chi}\right) & =\pi / 2.5: E\left(m_{\chi}\right)=20.847 \mathrm{keV} \frac{v^{2}}{c^{2}} \\
\alpha\left(m_{\chi}\right) & =\pi / 2.3: E\left(m_{\chi}\right)=26.029 \mathrm{keV} \frac{v^{2}}{c^{2}} \\
\alpha\left(m_{\chi}\right) & =\pi / 2: E\left(m_{\chi}\right)=31.177 \mathrm{keV} \frac{v^{2}}{c^{2}}
\end{aligned}
$$

In comparison, we also calculate an energy from expressions involving novel (sterile neutrino) particle limiting velocities (28.1)-(28.5) with fixed congruent angle of $\alpha\left(m_{\chi}\right)=\pi / 2.5$ which with (25) yields $z\left(m_{\chi}\right)=1.2955$. Then $m_{\chi} \simeq 12 \mathrm{keV} / \mathrm{c}^{2}$ and according to (28.1)-(28.5) yields the same energy for variety of squared limiting velocities:

$$
E\left(c_{1,2}^{2}\right)=E\left(\operatorname{Re} c_{1,2}^{2}\right)=E\left(\operatorname{Im} c_{1,2}^{2}\right)=E\left(c_{3}^{2}\right)=\frac{3 \sqrt{3} m_{\chi}}{2 z\left(m_{\chi}\right)}=20.847 \mathrm{keV} \frac{v^{2}}{c^{2}}
$$

Similar analyses one can perform for novel sterile neutrino momenta, but restricting to just the real ones $(6.1,2)$ and $(6.3)$.

The fact that through the bicubic equation limiting particle velocity formalism the real particle energy can be equally well evaluated from the complex, real or imaginary particle limiting velocity-squared expression, makes the particle energy exceptional and important,almost, a unique natural quantity, particularly as relations (5. 6) shows explicitly in succession numerically equal real values $E\left(c_{1,2}^{2}\right)=E\left(\operatorname{Re} c_{1,2}^{2}\right)=E\left(\operatorname{Im} c_{1,2}^{2}\right)=E\left(c_{3}^{2}\right)$ for each velocity-squared, complex $c_{1}^{2}$, complex $c_{2}^{2}$ and real $c_{3}^{2}$. What this shows that one should not automatically discard complex or imaginary quantities in physics as their contents may support real physical quantities, such as energy. The linear forms of novel particle limiting velocities are not less important as their comparisons to the velocity of light may show how unique the velocity of light really is.

Conclusion: The bicubic equation of particle limiting velocity formalism, with described examples, demonstrated possibilities of investigating variety of particle dynamical involvments. The newest one is that this formalism offers a general method, although not necessarily always simple, as to how an ordinary particle may become a novel or even dark matter particle. 


\section{References}

Adams, T. et al.. (2012). Measurement of the neutrino velocity with OPERA detector in the CNGS beam. JHEP, 093, ARXIV: $1212,1276$.

Eisberg, R. M. (1990). Fundamentals of Modern Physics. Springer, John Wiley \& Sons, p. 34.

Griffiths, D. (2008). Fundamentals of Modern Physics. Springer, John Wiley \& Sons, p. 89.

Ng, K. C. Y., Roach, B. M., Perez, K., Beacom, J. F., Horiuchi, S., Krivonos, R., \& Wik, D. R. (2019). New constraints on sterile neutrino dark matter from NuSTAR M31 observations. Physical Review D., 99, 083005. https://doi.org/10.1103/PhysRevD.99.083005

Rade, L., \& Westergren, B. (1999). Mathematics Handbook. Springer, p. 65. https://doi.org/10.1007/978-3-662-03556-6

Śoln, J. (2014). Theoretical particle limiting velocity from the bicubic equation: Neutrino example. hysics Essays, 27(3), 448, arXiv: 1403. 2683. https://doi.org/10.4006/0836-1398-27.3.448

Śoln, J. (2015). Particle limiting velocities from the bicubic equation derived from the Einstein's kinematics: PeV electron case. Applied Physics Research, 7(4),37. https://doi.org/10.5539/apr.v7n4p37

Śoln, J. (2016). Limiting velocities of primary, obscure and normal particles: Self-annihilating obscure particle as an example of dark matter particle. Applied Physics Research, 8(5), 1. https://doi.org/10.5539/apr.v8n5p1

Śoln, J. (2017). Connecting dark matter particles with the primary, obscure and normal particles through implicit causality. Applied Physics Research, 9(3), 1. https://doi.org/10.5539/apr.v9n3p1

Śoln, J. (2018). Positive and negative particle masses in the bicubic equation limiting particle velocity formalism. Applied Physics Research, 10(1), 14. https://doi.org/10.5539/apr.v10n1p14

Śoln, J. (2018). Similarities and differences between positive and negative particle masses in the bicubic equation limiting particle velocity formalism: positive or negative muon neutrino mass?. Applied Physics Research, 10(5), 40. https://doi.org/10.5539/apr.v10n5p40

Śoln, J. (2019). Formation of particle real energy in the bicubic equation limiting particle velocity formalism with possible applications to light dark matter. Applied Physics Research, 11(2), 22. https://doi.org/10.5539/apr.v11n2p92

Śoln, J. (2020). Complex limiting velocity exppressions as likely characteristics of dark matter particles. Applied Physics Research, 12(4), 107. https://doi.org/10.5539/apr.v12n4p107

Śoln, J. (2021). Real energy dark matter particles with mostly complex limiting velocities in either quadratic or linear forms. European Journal of Applied Physics, 3(1), 71. https://doi.org/10.24018/ejphysics.2021.3.1.46

Stecker, F. W. (2015). Limiting superluminal electron and neutrino velocity using 2010 Crab Nebula Flare and the Ice Cube PeV neutrino event. Astroparticle Physics, 56, 16-18, ARXIV: 1306, 6095. https://doi.org/10.1016/j.astropartphys.2014.02.007

\section{Copyrights}

Copyright for this article is retained by the author(s), with first publication rights granted to the journal.

This is an open-access article distributed under the terms and conditions of the Creative Commons Attribution license (http://creativecommons.org/licenses/by/3.0/). 